\title{
Rainfall drives hydrocephalus in East Africa
}

\author{
Clinical article
}

\author{
Steven J. Schiff, M.D., Ph.D., ${ }^{1,2}$ Sylvia L. Ranjeva, B.S., ${ }^{1}$ Timothy D. Sauer, Ph.D., ${ }^{3}$ \\ aNd BENJAMin C. WARF, M.D. ${ }^{4}$
}

${ }^{1}$ Center for Neural Engineering and Department of Engineering Science and Mechanics, and ${ }^{2}$ Departments of Neurosurgery and Physics, The Pennsylvania State University, University Park, Pennsylvania; ${ }^{3}$ Department of Mathematics, George Mason University, Fairfax, Virginia; and ${ }^{4}$ Department of Neurosurgery, and Program for Global Surgery and Social Change, Department of Global Health and Social Medicine, Harvard Medical School, Children's Hospital Boston, Massachusetts

\begin{abstract}
Object. Hydrocephalus is one of the most common brain disorders in children throughout the world. The majority of infant hydrocephalus cases in East Africa appear to be postinfectious, related to preceding neonatal infections, and are thus preventable if the microbial origins and routes of infection can be characterized. In prior microbiological work, the authors noted evidence of seasonality in postinfectious hydrocephalus (PIH) cases.

Methods. The geographical address of 696 consecutive children with PIH who were treated over 6 years was fused with satellite rainfall data for the same time period. A comprehensive time series and spatiotemporal analysis of cases and rainfall was performed.

Results. Four infection-onset peaks were found to straddle the twice-yearly rainy season peaks, demonstrating that the infections occurred at intermediate levels of rainfall.

Conclusions. The findings in this study reveal a previously unknown link between climate and a neurosurgical condition. Satellite-derived rainfall dynamics are an important factor in driving the infections that lead to PIH. Given prior microbial analysis, these findings point to the importance of environmental factors with respect to preventing the newborn infections that lead to PIH.
\end{abstract}

(http://thejns.org/doi/abs/10.3171/2012.5.PEDS11557)

KEY WoRDS
Africa $\bullet$ Ugandrocephalus $\bullet$ ventriculitis $\quad$ neonatal sepsis $\bullet$ weather

$\mathrm{H}$ YDROCEPHALUS is the most common neurosurgical disorder in children, with rates of 3-5 per 1000 live births in developed countries ${ }^{3}$ and estimates in sub-Saharan Africa of more than 100,000 cases per year. ${ }^{28}$ The majority of infant hydrocephalus cases in East Africa appear to be postinfectious,${ }^{27}$ related to preceding neonatal infections, and are thus preventable if the microbial origins and routes of infection can be better understood. Here we show that climate is an important factor in driving the infections that lead to PIH.

\section{Methods}

This research was performed under the oversight of the institutional review boards of The Pennsylvania State

Abbreviations used in this paper: $\mathrm{NOAA}=$ National Oceanic and Atmospheric Administration; $\mathrm{PIH}=$ postinfectious hydrocephalus.
University, CURE Children's Hospital of Uganda, and Children's Hospital Boston.

Signal processing was performed with Matlab (MathWorks, Inc.), and multitaper spectral analysis was performed with the open source Chronux algorithms (version 2.0, http://chronux.org). ${ }^{15}$ For spectral analysis, a window length of 3 years was used, and this window was moved in increments of 1 month to produce spectrograms and coherograms (we set the time-bandwidth product to 3 and the number of tapers to 5). Rainfall data were treated as a continuous process, and case data as point processes. When coherencies were calculated for rainfall versus case data, mixed continuous-point process algorithms were used (Chronux 2.0).

The phase of a cycle labels the cycle according to its peaks and troughs. Phases were assigned for rainfall data using a Hilbert transform. ${ }^{2}$ Prior to phase assignment, data for each district were zero-phase filtered (both forward and then backward so the filter did not distort the 
timing of the phases) by using a fifth-order Chebyshev filter centered on 2 cycles per year.

To characterize the nonuniformity of histograms of rainfall based on month of the year or phase of the rainy seasons, we used an information measure based on Shannon entropy $(S) .{ }^{25}$ The total entropy formula is as follows:

$S=-\sum_{i=1 \ldots n} p(i) \ln p(i)$,

where $p(i)=($ number of counts per bin i) $/ \mathrm{N}, N$ is the total number of counts in the histogram, and $n$ is the number of bins. The maximal entropy, which corresponds to the most disordered state, is when all bins are equal (each $p(i)$ $=1 / n$ ) and the histogram is flat. The maximal entropy is therefore $S_{\max }=\ln (n)$. Then the probability of deviation from nonuniformity is $\left(\mathrm{S}_{\max }-\mathrm{S}\right) / \mathrm{S}_{\max }(0$ when the histogram is flat), and the probability of significance of nonuniformity is therefore equal to $1-\left[\left(\mathrm{S}_{\max }-\mathrm{S}\right) / \mathrm{S}_{\max }\right]$, which constitutes a probability value in our histogram analysis.

We characterized the spatiotemporal patterns of both cases and rainfall by using a mode analysis typically used to characterize patterns in physical and biological systems that change with time. ${ }^{21}$ This modal analysis was performed as a principal orthogonal decomposition, ${ }^{12}$ implemented with singular value decomposition, by using the methods described by Schiff et al. ${ }^{22}$ The mean was retained in these samples, to preserve this important feature of average monthly rainfall and the fact that case numbers were all nonnegative in value (removing the case mean would have produced negative case numbers).

For cross-correlation analysis, statistical confidence bounds were calculated by forming 100 surrogate $^{23}$ climate time series by identifying a random time point in the time series (excluding the first and last 10 months) and swapping the series before and after the random point selected. The 100 randomly swapped time series form an ensemble of time series in which all short-term correlations between climate and cases will be destroyed, and twice the SD of the surrogate correlations form statistical bootstrap confidence limits. ${ }^{17}$ All correlations greater than these confidence limits are potentially significant, especially where consecutive significant excursions are seen. We report both the absolute value of the total correlation at 0 time lag when it is greater than the 2-SD confidence limit, as well as the more powerful sum of the total significant correlation at all lags in our analysis.

Geographical maps of the districts of Uganda were generated in The Pennsylvania State University Maps Library. We used Digital Charts of the World, a product of the Environmental Systems Research Institute, to produce a digital global positioning system grid overlay that we could match with our case location data, as shown in Fig. 1Aa.

Localized rainfall estimates were derived from a mixture of ground and satellite data, assimilated using the African Rainfall Estimation Algorithm (version 2.0) (RFE2.0) developed at the US NOAA Climate Prediction Center. ${ }^{7,11}$ Satellite-based remote sensing of the scattering of cloud layer particles in 2 microwave bands is obtained at 6-hour time intervals, along with cloud-top tempera- tures from the infrared band. The assimilation method uses maximum likelihood estimation to merge the satellite data with daily rain gauge readings at ground stations in Africa, inferring magnitude of rainfall at the specified grid points.

\section{Results}

In Uganda, almost all cases of infant hydrocephalus are treated at a single specialty hospital, where previous findings suggested seasonality in the microorganism spectrum from infants with $\mathrm{PIH} .{ }^{14}$

The birthdates and dates of the subsequent infection and surgical intervention were determined for 696 consecutive children with PIH over 6 years, from 2000 to 2005. The address of each patient was associated with one of the 77 districts within Uganda. Satellite rainfall data were extracted for the same time period, and data from the $0.1^{\circ} \times 0.1^{\circ}$ rainfall grid were averaged over each geographic district for each month.

A diagnosis of PIH was established based on the following criteria: 1) no history consistent with hydrocephalus at birth; and 2) either a history of a febrile illness (typically with seizures) preceding the onset of clinically apparent hydrocephalus or alternative findings such as imaging and endoscopic results indicative of prior ventriculitis. ${ }^{27}$ In the vast majority $(76 \%)$ of such patients, the onset of their febrile illness resulting in PIH occurs within the 1st month of life-consistent with neonatal sepsis. ${ }^{27}$ In the present study, the mean and median ages of children presenting for hydrocephalus treatment were 9.1 and 4 months, respectively, due to the fraction of children who presented later with more advanced untreated hydrocephalus. Because most infants with PIH in sub-Saharan Africa have no access to the neurological surgery required to palliate this condition, and their brains are often severely damaged as a consequence of the inciting infection, the most effective approach in such children will be prevention. ${ }^{14}$

The NOAA collects satellite data from multiple satellites, and the African Rainfall Estimation Algorithm generates rainfall estimates beginning with March 2000 . $^{71}$ We fused 3600 grid points of these spatiotemporal data with the geography of Uganda (Fig. 1A), by averaging rainfall per month temporally and within each of 77 Ugandan districts spatially. We formed a $10 \times 10$ adjacency matrix preserving at least 1 or more district adjacencies to facilitate display of spatiotemporal results (Fig. 1Ab).

Uganda and the East African highlands have 2 rainy seasons per year. Although the amplitudes of monthly rain vary, the timing of these seasonal cycles is very regular across districts (Fig. 1Ba). A spectrogram applied to such data reveals a strong fundamental frequency at 2 cycles per year (Fig. 1Ba). Case numbers by date of birth (696 cases), of febrile illness onset (692 cases), and of surgical intervention (632 cases), and the frequency content of such data are shown in Fig. $1 \mathrm{Bb}-1 \mathrm{Bd}$. There were fewer dates of surgery than for birth dates, because in some admissions the patients were operated on in the months following 2005, some died prior to surgery, and in some 
$\mathrm{Aa}$

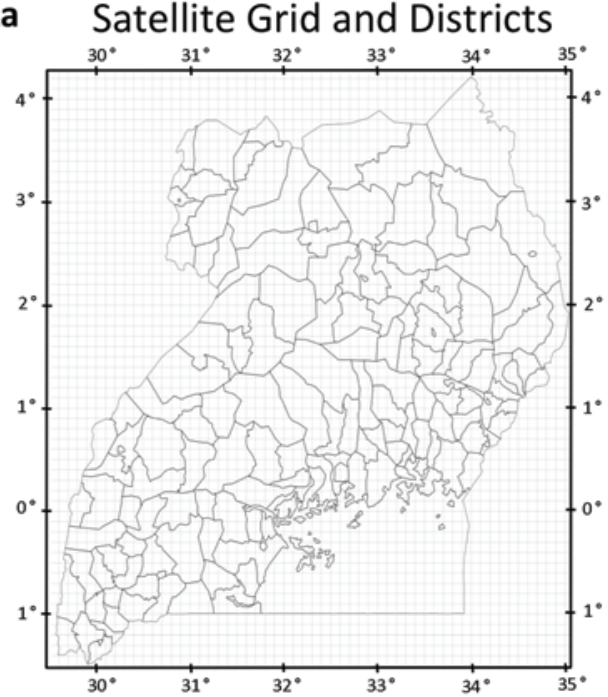

Ab

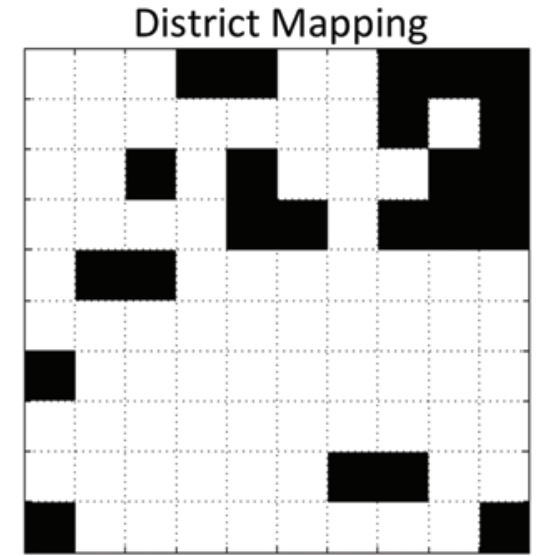

$\mathrm{Ba}_{400}$
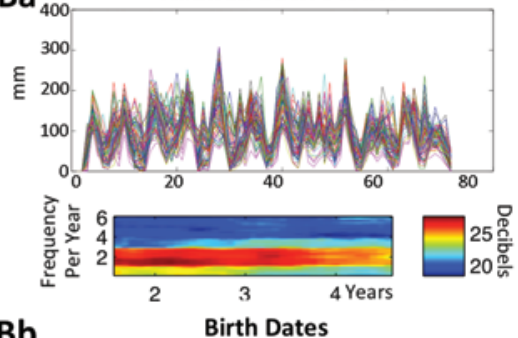

$\mathrm{Bb}$
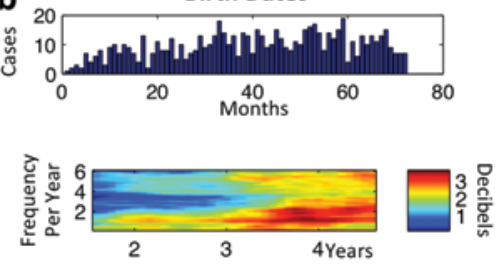

$\mathrm{Bc}$
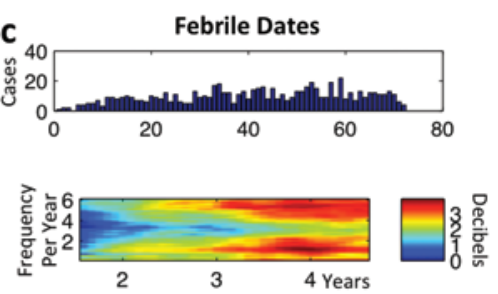

Bd
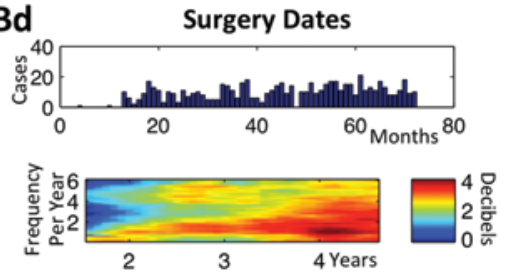

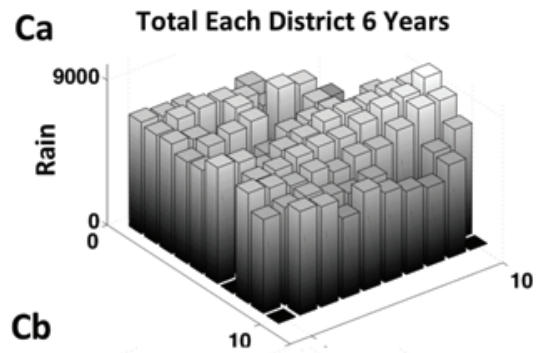

6

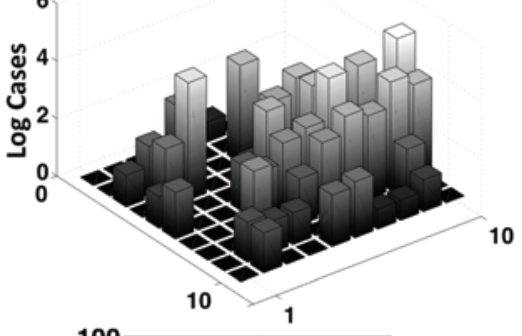

Cc
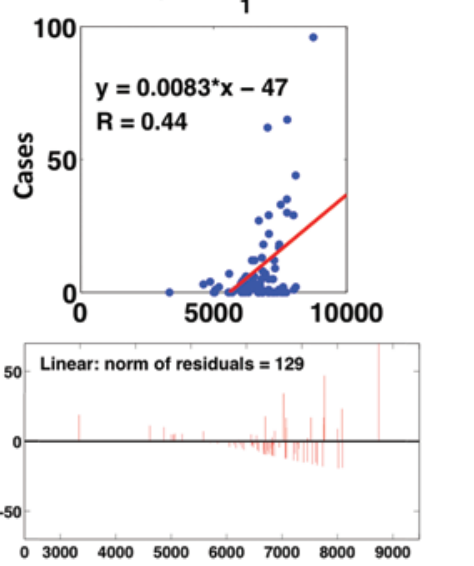

Total Rainfall Each District 6 Years

Fig. 1. Panel Aa shows a satellite $0.1^{\circ} \times 0.1^{\circ}$ grid overlain on the geographic districts of Uganda. Monthly rainfall was averaged within each district, and patients with $\mathrm{PIH}$ were assigned to a district. Satellite data are missing for January and February of 2000 , and these data were set to 0 . These 77 districts were mapped to a $10 \times 10$ adjacency matrix in panel $\mathbf{A b}$, keeping at least 1 or more neighboring districts in contact to facilitate display of spatiotemporal analysis. The 23 black squares are empty. The rainfall by month from each district is shown overlain in panel $\mathbf{B a}$, and the spectrogram demonstrates a strong twice-yearly frequency of rainy seasons. Birth, febrile illness, and surgery dates are shown in panels $\mathbf{B b}, \mathbf{B c}$, and $\mathbf{B d}$, respectively, and their spectrograms are complex. Note that the time axes for the spectrograms are shortened to 1.5-4.5 years, reflecting the resolution within the 3 -year sliding windows used. Summing the total rainfall (in $\mathrm{mm}$ ) for each district over all 6 years reflects the high levels of rainfall across Uganda in panel $\mathrm{Ca}$ and the more complex spatial distribution of cases over all 6 years in panel $\mathrm{Cb}$ (a base $10 \log$ scale is used for cases to facilitate the display). A linear regression of total rainfall to cases (using febrile illness dates, for comparison later) in panel $\mathrm{Cc}$ demonstrates a modest fit (coefficient of determination, $R^{2}=0.44$ ), and the residuals of this fit steadily increase as total rainfall increases, indicating that the relationship between rain and cases over geography is not a simple one explained by total rainfall. A regression by birth dates is nearly identical $\left(R^{2}=0.43\right)$.

the disease was managed without surgery. The frequency content of cases was more complex than that of rainfall. Looking at spatial variations by summing over time, we see that there is a great deal of rainfall over the 6 years in Uganda (Fig. 1Ca) and that the relationship of cases to total rainfall is complex (Fig. 1Cb). Although there is a modest linear fit of cases per district to rainfall (linear regression coefficient of determination, $\mathrm{R}^{2}=0.44$ ), the residuals of this fit steadily increase as rainfall increases. This confirms that the relationship between rain and cases over geography is not a simple linear one explained by total rainfall.

We averaged the rainfall per month across all districts, and summed the cases per month, to produce average global univariate time series (Fig. 2A and B). We found that the cross-correlation between rainfall and cases is stronger for febrile illness and birth dates than for surgical dates. Cross-correlation is a function of the time lag between 2 signals, and although the cross-correlation at 0 time lag is often used to quantify an interaction, when there are delays between cause and effect (as in infection and postinfectious consequences), one can sum the cross-correlation over a range of lags. ${ }^{23}$ We used the level of statistical confidence (see legend to Fig. 2) to select the time lag ranges to use to sum only the significant cross-correlation values. ${ }^{23}$ The febrile case dates have more than twice the 


\section{Average Global Time Series}

A
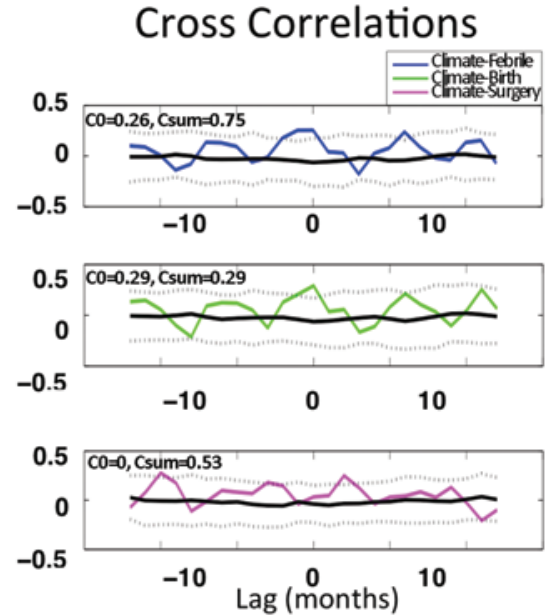
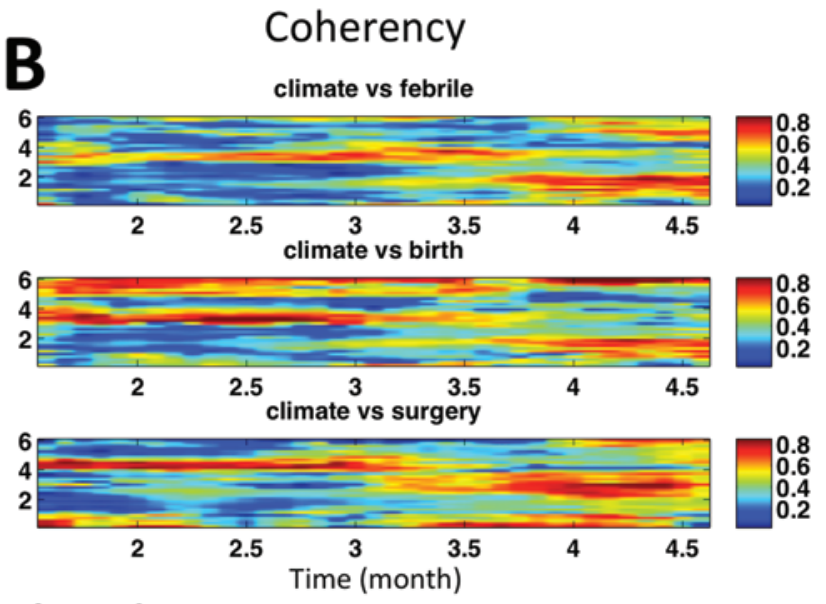

\section{Spatiotemporal Modes}

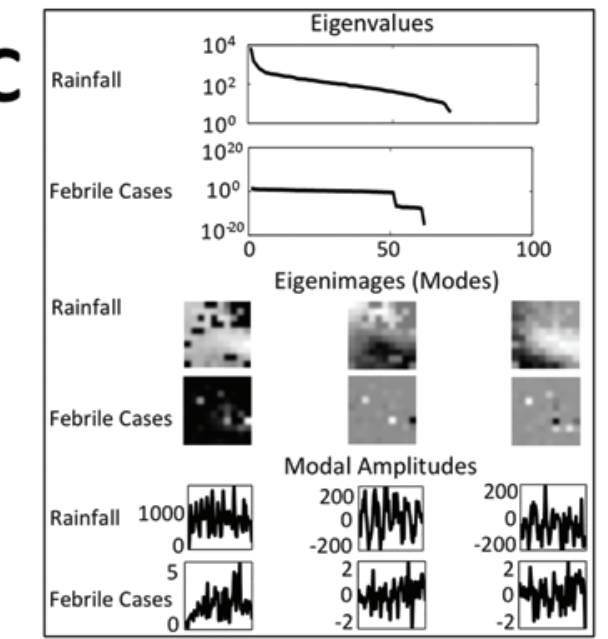

D

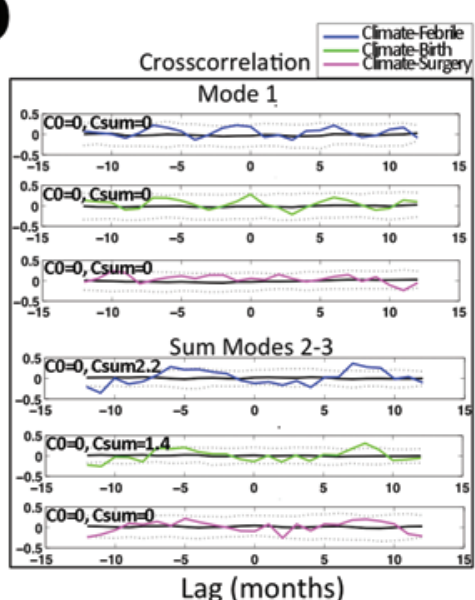

$\mathbf{E}$

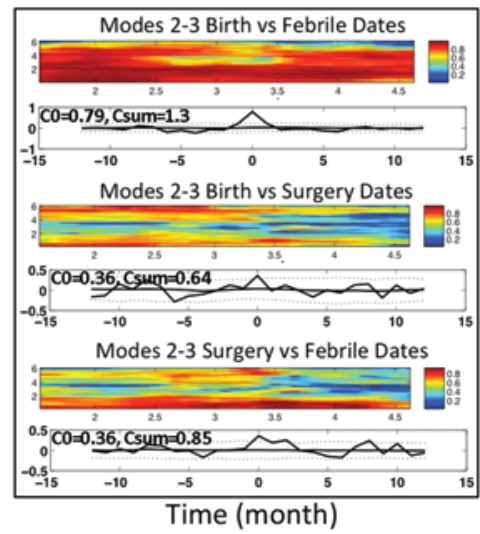

Fig. 2. Average global time series (univariate) are shown in panels $\mathbf{A}$ and $\mathbf{B}$, and spatiotemporal analysis in panels $\mathbf{C}-\mathbf{E}$. After averaging rainfall and summing cases across districts by month, their cross-correlations are shown in panel $A$. Confidence bounds were calculated as described in Methods, representing twice the SD of surrogate correlations displayed as the dotted line bootstrap confidence limits in panel A. All correlations greater than these confidence limits are potentially significant, especially where consecutive significant excursions are seen. The correlation with rainfall is greatest for febrile illness dates, and the surgery dates are offset from febrile illness and birth dates by several months, as expected. The coherograms of the data from panel $A$ are shown in B, where a prominent band at 4 cycles per year emerges from each of the data sets. The full data sets for each district were decomposed using a singular value decomposition, and the eigenvalues (statistical importance of the eigenimages) and eigenimages (most statistically common pattern to the data, ordered by the eigenvalues from largest to smallest) are shown for rainfall and febrile illness dates in panel $\mathrm{C}$. There is a prominent first mode, which represents the average total rainfall or case numbers, followed by second and third modes displaying fluctuations about the mean. These modes were used as filters to form reconstructed univariate time series in panel $\mathrm{D}$ (products of eigenvalue, modal amplitude, and eigenimage, and summing to reconstruct data), where it is seen that there were no correlations between the first rainfall mode and case data. Testing all combinations of the first 6 modes, we find the largest correlations by summing modes 2 and 3 , where febrile illness dates demonstrate larger correlations with rainfall than birth dates, and no correlation in these modes is seen for surgery dates. In panel $\mathrm{E}$ we show coherograms from the sum of modes 2 and 3 , demonstrating that there is very substantial correlation between birth and febrile illness dates and that febrile illness dates correlate more strongly with surgery dates (in the correlation sums at arbitrary lags [Csum]) than do the birth dates. $\mathrm{CO}=$ absolute value of the total correlation at 0 time lag when greater than the 2-SD confidence limit; Csum = sum of the total significant correlation at all lags.

significant summed cross-correlation than that of the birth dates (correlation sum 0.75 vs 0.29 ), and they are also both significantly peak correlated around the 0-month time lag. The surgical cases have a peak time lag offset by several months, consistent with the previously reported time from illness to onset of noticeable hydrocephalus (mean 0.72 months) and the delay between hydrocephalus onset and presentation for surgical treatment (mean 7.46 months). ${ }^{3}$ Using these same averaged univariate data, we performed mixed point-process and continuous coherence calculations in which the Chronux algorithms (http://chronux. org/) were used. These coherograms each demonstrated a 
4-cycle-per-year coherency (Fig. 2B) that requires explanation.

A spatiotemporal analysis in which a mode decomposition was used was performed using methods described by Schiff et al..$^{22}$ The first mode represents the statistically most likely pattern given each monthly spatial data snapshot in time. The first mode for both rainfall and cases retains the monthly average, and the time series of this mode is reflected in the modal amplitudes (Fig. 2C). Note that the modal amplitudes of the first mode for both rainfall and cases are all positive (there are no negative rainfalls or cases) and that the subsequent modal amplitudes fluctuate around zero. We use the Schmidt approximation theorem ${ }^{24}$ to form optimal low-order approximations to the dynamics. We filter the data by removing the first mode with its running average rainfall, ${ }^{16}$ and we then sum 1 or more of the remaining modes together (summing all of the modes would return the original data set). Because the first mode shows no correlation with cases (Fig. 2D), this confirms that the average monthly rainfall is not the important factor here (consistent with the regression result of Fig. 1C). However, if we remove this first mode, very substantial correlation with cases is observed in the subsequent modes. Eliminating the first mode, we then find that, of all possible partial sums of modes 2-6, the strongest correlations between rainfall and cases are seen in the sum of modes 2 and 3, demonstrating far more substantial correlations with rainfall than shown from the raw data in Fig. 2A for febrile illness and birth dates (significant correlation sums 2.2 and 1.4 for modal filtered, vs 0.75 and 0.29 for averages of raw febrile and birth dates). For data filtered through the modes in this manner, birth versus febrile illness correlations and coherencies are very strong, but febrile illness dates correlate more strongly with surgery dates than birth dates, as we would expect clinically (Fig. 2E).

Returning to the raw data, we next found that the cases occurred at intermediate levels of rainfall, with a very strong Gaussian distribution (Fig. 3A). The mean rainfalls for febrile illness and birth dates were very close, whereas surgical dates were different, reflecting the offset in months until surgery. Average rainfall per month for all 6 years is shown in Fig. 3Ba, and beneath that, the total cases per month for all 6 years based on febrile illness, birth, and surgery month. Each case distribution significantly deviates from uniform, demonstrating a monthly dependence, with the most nonuniform distribution for febrile cases $(p=0.0066$ according to Shannon entropy). Note that the febrile case months have 4 peaks (March, May, September, and November), which flank the peak rainfall months (April and October). These 4 peaks appear to explain the 4-cycle per year coherency bands in Fig. 2B.

However, it is rainfall, rather than month, that is the key element to consider relating rainfall and cases. The rainy seasons are so regular in Uganda that one could often assign a phase reasonably accurately by month for average monthly data. Nevertheless, for individual districts, and for individual years, it is more accurate to assign a phase for each district rigorously. We therefore narrowband filtered each district's rainfall data by using a filter that does not distort the timing of the phases (centered at $2 \mathrm{~Hz}$; Fig. 3Ca), and the phases for each district were assigned from 0 to $4 \pi$ to cover each consecutive 2 rainy seasons (Fig. 3Cb). The average rainfall per phase is fit with a sine wave in Fig. 3D, and beneath that plot are shown the histograms of case numbers per phase. Again, we see the strong pattern of febrile illness cases, with 2 peaks in phase flanking each peak in rain (now dissociated from month of occurrence). This is consistent with the evidence in Fig. 3A that the illnesses occurred at intermediate levels of rainfall.

\section{Discussion}

Our findings demonstrate a strong correlation between satellite-determined geographic rainfall dynamics and a childhood disease that previously had no known link to environment and climate.

Climate dynamics driving malaria cases in the East African highlands have been shown to be linked to increased rainfall and are dynamically linked to rainfall fluctuations caused by the El Niño-Southern Oscillation and the intensity of the Indian Ocean dipole surface temperature gradient. ${ }^{8,9}$ Environmental conditions including rainfall ${ }^{5,6}$ and soil hydrology $y^{26}$ have been shown to be important in the acquisition of Burkholderia pseudomallei infections in Southeast Asia and Australia, and the epidemiological rainfall interactions for this organism can be complex and vary by geographical region. $.^{10} \mathrm{Me}-$ ningococcal meningitis is the cause of seasonal dry-season epidemics in regions of sub-Saharan Africa north of Uganda, ${ }^{18}$ and such cases can produce PIH. ${ }^{1}$

The closest parallel to our findings, where peaks in case incidence are split around the peaks in rainfall, are seen in patterns of cholera in both India ${ }^{19}$ and Bangladesh. ${ }^{13,20}$ Although cholera dynamics are complex, there appears to be a quenching of the infectivity of waterborne secondary infections when peak rainfall dilutes the pathogenic concentrations. It is possible that a similar environmental mechanism serves to quench our case numbers of neonatal sepsis during peak rainfall months.

Previous work demonstrated seasonal evidence for genetic linkages between bacterial DNA obtained in infants with PIH at the time of surgery and environmental samples, along with evidence relating these organisms to factors of domestic animals and hut construction. ${ }^{14}$ Infants typically present with PIH several months after neonatal sepsis, and cultures from more than 1000 consecutive cases of PIH yielded almost uniformly negative results. ${ }^{27}$ Using sequencing for bacterial ribosomal $16 \mathrm{~S}$ DNA remaining in such patients revealed amplifiable sequences from nearly all children's CSF recovered sterilely during surgery, a predominance of gammaproteobacteria (a class of gram-negative organisms), and similar genetic matches with environmental samples from the infants' village environments. ${ }^{14}$ Such findings demonstrate the association of such infants with microorganisms from their village environments, but do not isolate the causative organisms of neonatal sepsis that lead to PIH. A large-scale study of the bacterial and viral components associated with neonatal sepsis in this neonatal population is underway. A simultaneous strategy of both improving the antimicro- 

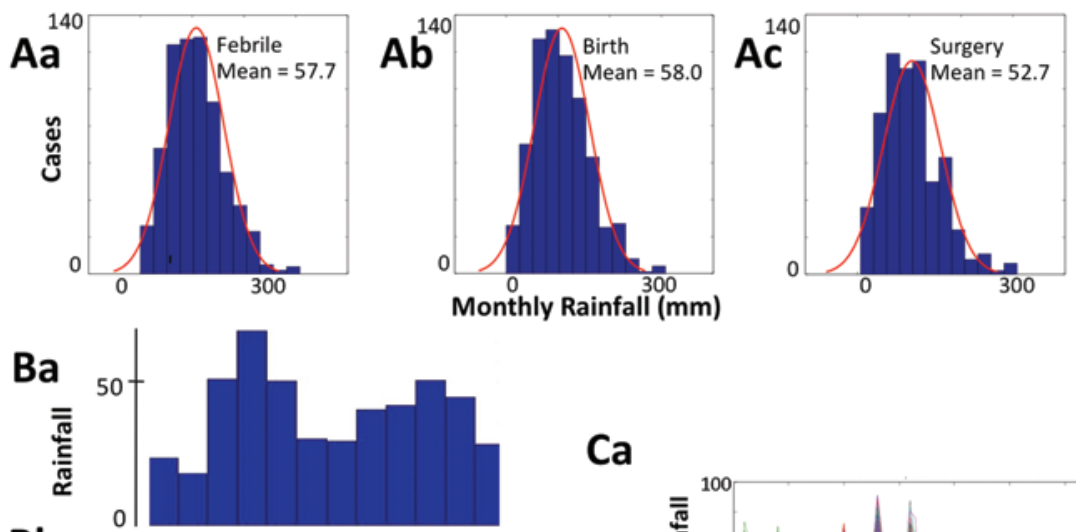

Bb

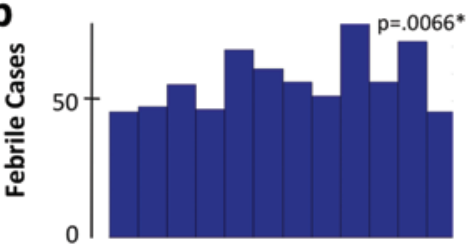

Bc
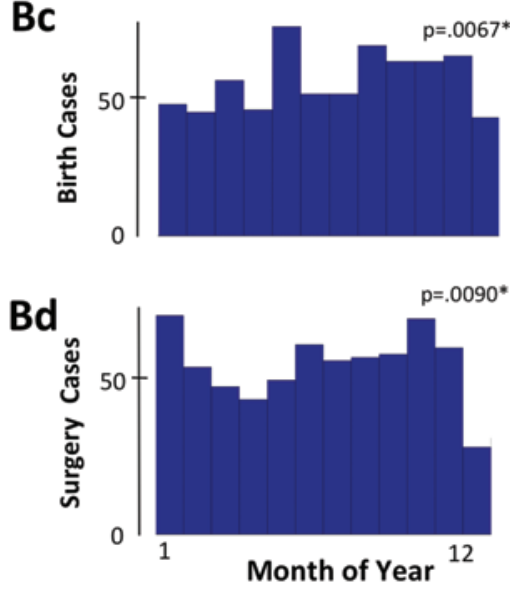

Ca

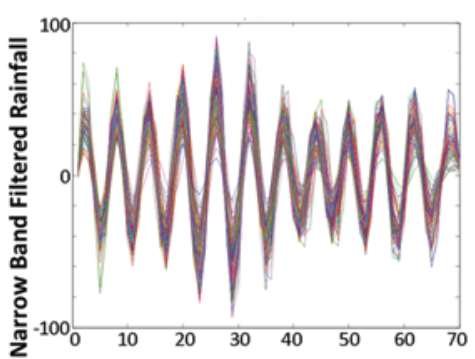

$\mathrm{Cb}$

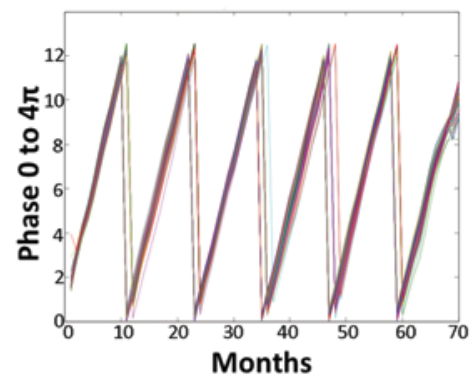

Da

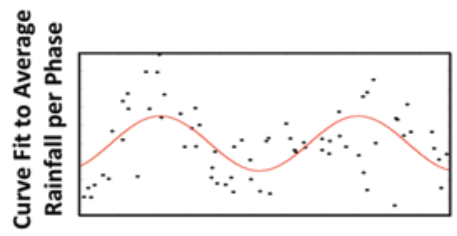

Db

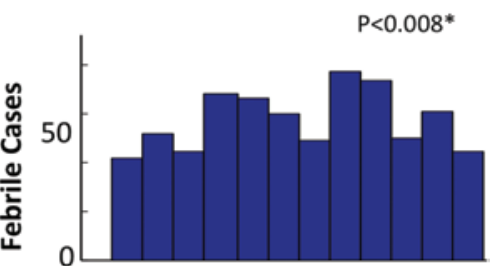

Dc

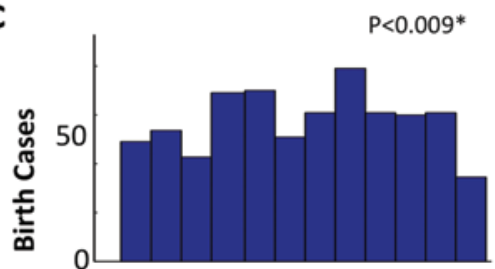

Db

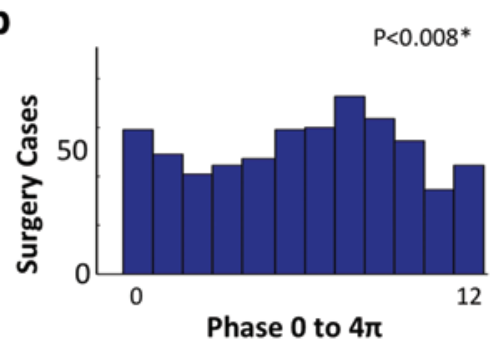

Fig. 3. Histograms for all district case data per month (5544 samples) shown as a function of rainfall per month for febrile illness, birth dates, and surgery dates in panels Aa-Ac. The cases are strongly peaked at intermediate levels of rainfall. Normal distribution fits are shown, which indicate that the birth and febrile illness months have almost identical means, whereas the surgery mean rainfall values are offset. A histogram of monthly rainfall is shown in panel $\mathbf{B a}$, along with monthly cases based on febrile illness, birth, and surgery dates in panels $\mathbf{B b}-\mathbf{B d}$. Tests of nonuniformity based on Shannon entropy show that each case histogram is significantly nonuniform with respect to monthly rainfall, with febrile dates being the most nonuniform $(p=0.0066)$. Note the 4 peaks per year in monthly febrile cases flanking the 2 peaks in rainfall in April and October. Rainfall for each district was then narrow-band filtered from 1.5 to $2.5 \mathrm{~Hz}$ by using a fifth-order Chebyshev filter applied so as not to distort phase in panel $\mathrm{Ca}$, and a Hilbert transform was used to assign phase from 0 to $4 \pi$ to represent yearly cycles in panel $\mathrm{Cb}$. The average rainfall per phase was fit by a sine wave in panel $\mathrm{Da}$, and the histograms of case data are shown in panels $\mathrm{Db}-\mathrm{Dd}$. Again, assigning phase values rather than calendar months demonstrates that febrile illness occurs with 4 peaks per year, straddling the phase peaks, which coincide with peak rainfall months.

bial therapy of neonates infected with these neurotropic agents and engineering rational environmental health strategies to prevent these infections will be required.

Fusing spatiotemporal dynamics from satellite and case data are readily adaptable to a wide variety of infectious disease processes for which complex interactions with the environment may exist. In diseases in which the establishment of rainfall as a factor is clear, ${ }^{9,10}$ understanding unexplained geographical variability would be aided by a spatiotemporal approach. Furthermore, as climate change gathers increasing international focus, a strategy such as we have presented opens the prospect of observing and predicting the influence of changing atmospheric dynamics in driving infectious disease patterns with an increased degree of spatial granularity than is otherwise possible.

\section{Conclusions}

At present, PIH is treated neurosurgically-an expensive and complex level of medical intervention-albeit one whose cost-effectiveness can be readily demonstrated. ${ }^{28}$ Prevention of PIH will require an understand- 
ing of the spectrum of organisms and routes of infection that lead to the particular cases of neonatal sepsis which predispose to PIH. Our findings here are helpful in ongoing efforts to identify both the agents involved and their routes of infection. Although medically sophisticated approaches to improving the predictive pharmacological treatment of infants presenting with neonatal sepsis may be derived from a fusion of climate and microbiological models, the optimal approach to reducing such diseases of impoverishment will lie with unraveling the environmental mechanisms and interrupting them. ${ }^{4}$

\section{Disclosure}

This work was supported by the Penn State Clinical Translational Sciences Institute and the endowment funds of Harvey F. Brush. The authors report no conflict of interest concerning the materials or methods used in this study or the findings specified in this paper.

Author contributions to the study and manuscript preparation include the following. Conception and design: Schiff, Warf. Acquisition of data: Schiff, Warf. Analysis and interpretation of data: Schiff, Ranjeva, Sauer. Drafting the article: Schiff, Sauer, Warf. Critically revising the article: all authors. Reviewed submitted version of manuscript: all authors. Approved the final version of the manuscript on behalf of all authors: Schiff. Statistical analysis: Schiff, Ranjeva, Sauer. Study supervision: Schiff.

\section{Acknowledgment}

The authors appreciate the helpful discussions with S. Peacock.

\section{References}

1. Aziz IA: Hydrocephalus in the Sudan. J R Coll Surg Edinb 21:222-224, 1976

2. Bendat JS, Piersol AG: Random Data: Analysis and Measurement Procedures, ed 2. New York: Wiley, 1986, pp 484-516

3. Chi JH, Fullerton HJ, Gupta N: Time trends and demographics of deaths from congenital hydrocephalus in children in the United States: National Center for Health Statistics data, 1979 to 1998. J Neurosurg 103 (2 Suppl):113-118, 2005

4. Colwell RR, Huq A, Islam MS, Aziz KMA, Yunus M, Khan $\mathrm{NH}$, et al: Reduction of cholera in Bangladeshi villages by simple filtration. Proc Natl Acad Sci U S A 100:1051-1055, 2003

5. Corkeron ML, Norton R, Nelson PN: Spatial analysis of melioidosis distribution in a suburban area. Epidemiol Infect 138:1346-1352, 2010

6. Currie BJ, Jacups SP: Intensity of rainfall and severity of melioidosis, Australia. Emerg Infect Dis 9:1538-1542, 2003

7. Ferraro RR, Grody NC, Weng F, Basist A: An eight-year time series of rainfall, clouds, water vapor, snow cover, and sea ice derived from SSM/I measurements. Bull Am Meteorol Soc 77:891-906, 1996

8. Hashizume M, Chaves LF, Minakawa N: Indian Ocean Dipole drives malaria resurgence in East African highlands. Sci Rep 2:269, 2012

9. Hashizume M, Terao T, Minakawa N: The Indian Ocean Dipole and malaria risk in the highlands of western Kenya. Proc Natl Acad Sci U S A 106:1857-1862, 2009

10. Hassan MR, Pani SP, Peng NP, Voralu K, Vijayalakshmi N, Mehanderkar R, et al: Incidence, risk factors and clinical epidemiology of melioidosis: a complex socio-ecological emerging infectious disease in the Alor Setar region of Kedah, Malaysia. BMC Infect Dis 10:302, 2010
11. Herman A, Kumar VB, Arkin PA, Kousky JV: Objectively determined 10-day African rainfall estimates created for famine early warning systems. Int J Remote Sens 18:2147-2159, 1997

12. Holmes P, Lumley JL, Berkooz G: Turbulence, Coherent Structures, Dynamical Systems, and Symmetry. Cambridge: Cambridge University Press, 1996

13. Koelle K, Rodó X, Pascual M, Yunus M, Mostafa G: Refractory periods and climate forcing in cholera dynamics. Nature 436:696-700, 2005

14. Li L, Padhi A, Ranjeva SL, Donaldson SC, Warf BC, Mugamba J, et al: Association of bacteria with hydrocephalus in Ugandan infants. Clinical article. J Neurosurg Pediatr 7: 73-87, 2011

15. Mitra P, Bokil H: Observed Brain Dynamics. New York: Oxford University Press, 2008

16. Mitra PP, Pesaran B: Analysis of dynamic brain imaging data. Biophys J 76:691-708, 1999

17. Netoff TI, Carroll TL, Pecora LM, Schiff SJ: Detecting coupling in the presence of noise and nonlinearity, in Schelter B, Winterhalder M, Timmer J (eds): Handbook of Time Series Analysis. Weinheim: Wiley-VCH Verlag GmbH \& Co. KGaA, 2006, pp 265-282

18. Roberts L: Infectious disease. An ill wind, bringing meningitis. Science 320:1710-1715, 2008

19. Ruiz-Moreno D, Pascual M, Bouma M, Dobson A, Cash B: Cholera seasonality in madras (1901-1940): dual role for rainfall in endemic and epidemic regions. EcoHealth 4:52-62, 2007

20. Ruiz-Moreno D, Pascual M, Emch M, Yunus M: Spatial clustering in the spatio-temporal dynamics of endemic cholera. BMC Infect Dis 10:51, 2010

21. Schiff SJ: Neural Control Engineering. Cambridge: MIT Press, 2012

22. Schiff SJ, Huang X, Wu JY: Dynamical evolution of spatiotemporal patterns in mammalian middle cortex. Phys Rev Lett 98:178102, 2007

23. Schiff SJ, Sauer T, Kumar R, Weinstein SL: Neuronal spatiotemporal pattern discrimination: the dynamical evolution of seizures. Neuroimage 28:1043-1055, 2005

24. Schmidt E: Zur Theorie der linearen und nichtlinearen Integralgleichungen. I. Teil: Entwicklung willkürlicher Funktionen nach Systemen vorgeschriebener. Math Ann 63:433476, 1907

25. Tass P, Rosenblum MG, Weule J, Kurths J, Pikovsky A, Volkmann J, et al: Detection of n:m phase locking from noisy data: application to magnetoencephalography. Phys Rev Lett 81:3291-3294, 1998

26. Thomas AD, Forbes-Faulkner J, Parker M: Isolation of Pseudomonas pseudomallei from clay layers at defined depths. Am J Epidemiol 110:515-521, 1979

27. Warf BC: Hydrocephalus in Uganda: the predominance of infectious origin and primary management with endoscopic third ventriculostomy. J Neurosurg 102 (1 Suppl):1-15, 2005

28. Warf BC, Alkire BC, Bhai S, Hughes C, Schiff SJ, Vincent J, et al: Infant hydrocephalus in sub-Saharan Africa: Cost and benefit of neurosurgical intervention. Clinical article. J Neurosurg Pediatr 8:509-521, 2011 (Erratum in J Neurosurg Pediatr 9:109, 2012)

Manuscript submitted December 20, 2011.

Accepted May 14, 2012.

Please include this information when citing this paper: published online July 6, 2012; DOI: 10.3171/2012.5 .PEDS11557.

Address correspondence to: Steven J. Schiff, M.D., Center for Neural Engineering, W311 Millennium Science Complex, Penn State University, University Park, Pennsylvania 16802. email: sschiff@psu.edu. 